\title{
Quantitative EELS by Spectrum Parametrization
}

\author{
Abdelaziz Aitouchen, Yolande Kihn and Gérald Zanchi \\ Centre d'Élaboration des Matériaux et d'Études Structurales, 29 rue J. Marvig, BP 4347, \\ 31055 Toulouse Cedex 04, France
}

(Received August 12, 1997; accepted February 13, 1998)

PACS.29.30.- $\mathrm{h}$ - Spectrometers and spectroscopic techniques

PACS.61.14.- $\mathrm{x}$ - Electron diffraction and scattering

\begin{abstract}
In order to describe the profiles of electron energy loss spectra in transmission electron microscopy, an analytical form of the intensity has been established taking the various elementary diffusion processes into account. This procedure is used to evaluate the background profile in each part of the spectra, even for thick samples. Simple diffusion models can then be established from experimental spectra and the application area of quantitative elementary chemical analysis can hence be extended.
\end{abstract}

Résumé. - Dans le but de décrire les profils de spectres de pertes d'énergie d'électrons en microscopie électronique en transmission, une expression analytique de l'intensité a été établie en tenant compte des divers processus de diffusion élémentaires. L'un des intérêts majeurs de cette méthode de calcul est de pouvoir évaluer le fond continu en tout point du spectre, quelle que soit l'épaisseur de l'échantillon analysée. Ceci permet d'une part de définir les modèles de diffusion simple à partir des spectres expérimentaux et d'autre part d'élargir le domaine d'application de l'analyse chimique élémentaire quantitative.

\section{Introduction}

In Electron Energy-Loss Spectroscopy (EELS), chemical analysis is quantified by using the approximate formula that relates the number $N$ of atoms per unit area of specimen to the characteristic-loss signal measured as the area $I_{\mathrm{c}}(\beta, \Delta)$ integrated over an energy range $\Delta$ beyond the ionization edge and over the angular range $\beta$ of scattering collected by the spectrometer [1]:

$$
I_{\mathrm{c}}(\beta, \Delta) \approx N \sigma_{\mathrm{c}}(\beta, \Delta) I_{1}(\beta, \Delta)
$$

Here $\sigma_{\mathrm{c}}(\beta, \Delta)$ is the partial cross-section for inner-shell scattering, and $I_{1}(\beta, \Delta)$ is the low-loss intensity integrated up to $E=\Delta$. When only elemental ratios are required, $I_{1}(\beta, \Delta)$ is the same for each element and cancels out in the calculation of atomic ratios.

Various factors affect the accuracy of elemental analysis $[2,3]$. The above equation involves some approximations, particularly due to the influence of elastic and low-energy inelastic scattering. The procedural errors can be minimized by using thin samples, and deconvolution if plural scattering is important [3-5]. 
Apart from the validity limits of the initial equation, the accuracy of chemical analysis depends on the calculation of inner-shell scattering cross-sections and the measurement of characteristic signals.

\section{Cross-Section Evaluation}

The most widely used method of calculating partial cross-sections is the hydrogenic model, introduced by Egerton [6]. More recently, it has been shown that the accuracy of the crosssection calculations is improved by introducing the Hartree-Slater model and oscillator-strength parametrization [7-9]. The "SIGMAK" and "SIGMAL" revised programs [10] allow the partial cross-sections to be calculated with good accuracy, at least for low $Z$ elements or when the edges involved are not separated by a large energy range.

\section{Characteristic Signal Measurement}

A characteristic signal in a spectrum is defined as the area delimited by the peak and the background, evaluated over an energy range $\Delta$ beyond the edge. One of the main problems that arises during the quantitative exploitation of EELS data is the extraction of the characteristic core-loss signals, which are superposed on an atypical and monotonically decreasing background, originating from plasmon plural scattering or from the overlap of other core-loss distributions.

In quantitative elemental analysis, the core-loss distributions are isolated by straightforward subtraction of the background intensity under the edges. This background removal procedure is generally performed by an extrapolation method proposed by Egerton [2]. The pre-edge region is first fitted to an $A E^{-r}$ power law and extrapolated beyond the edge. The fit is then subtracted from the total spectrum intensity to give the core-loss signal. The form $A E^{-r}$ is widely used because it contains only two parameters to be adjusted and it yields a good fit in many cases. Several ways of adjusting the parameters $A$ and $r$ are commonly used: the 2-area method [2], ravine-search [11] and simplex [12,13] procedures. Some variants of the power-law have been tested, particularly for low-energy edges $[14,15]$. The extrapolation technique fails in the case of overlap of the edges and gives poor results when the core-loss signal is weak and for thick samples. In order to overcome these difficulties, standard edge and convolution techniques $[16,17]$ have been used in low concentration quantifications. Another quantitative approach consists in modelling the spectrum before and after an excitation edge by considering that each edge can be described as the sum of different contributions [18-20]. This method is effective in the quantification of overlapping edges.

In the case of relative thick samples, the plural scattering effects increase inducing two problems: the form $A E^{-r}$ is not convenient to represent the background and the lower energy edge can be inclosed in the background due to the convolution effect, as shown in boron nitride $[21,22]$ where the boron $K$-edge becomes not visible.

The focus of this work is to provide an alternative to the classical signal stripping methods by computing the entire profiles of the electron energy loss spectra, The method takes into account such experimental parameters as the specimen thickness, the acceptance angle and the transfer function of the spectrometer.

It is now well established [23-25] that energy loss spectra result from multiple scattering of the electrons according to a limited number of basic elementary processes, typically plasmon or single electron excitations. 


\section{Calculation of a Spectrum}

The intensity of the transmitted beam can be written as a function of the energy loss $E$ taking the electron angular scattering $\theta$ into account. The spectrum intensity is calculated assuming that the probability for an electron to suffer an energy loss $E$ and to be deviated through an angle $\theta$ after an interaction of any kind is given by a normalized function, which can be written as a product of two normalized functions $g(E)$ and $f(\theta)$ [26]. Assuming in addition that the excitation probabilities for any process obey a Poisson law, the beam intensity can be expressed in Fourier space as follows:

$$
\hat{I}\left(w, \theta_{\mathrm{d}}, \zeta\right)=\hat{I}_{0}\left(w, \theta_{\mathrm{d}}\right) \hat{J}(w, \zeta) .
$$

In this expression, $w$ is the reciprocal variable corresponding to the energy loss $E, \theta_{\mathrm{d}}$ is the collection angle of the spectrometer, $\zeta=t / \lambda_{1}$ is the reduced thickness, $\lambda_{1}$ being the mean free path associated with the main inelastic process (generally a bulk plasmon) and $t$ the thickness of the sample. The function $\hat{J}(w, \zeta)$ is defined by:

$$
\hat{J}(w, \zeta)=\hat{G}_{\mathrm{s}}(w) \exp \left\{\zeta\left(\hat{g}_{1}(w)\right)+\sum_{i=2}^{n} \frac{\lambda_{1}}{\lambda_{i}}\left(\hat{g}_{i}(w)\right)\right\} .
$$

$\hat{G}_{\mathrm{S}}(w), \hat{g}_{1}(w)$ and $\hat{g}_{i}(w)$ are the Fourier transforms of the functions $G_{\mathrm{S}}(E), g_{1}(E)$ and $g_{i}(E)$. The first of these, $G_{\mathrm{s}}(E)$ represents the transfer function of the whole experimental system. This function takes the energy fluctuations of the incident beam as well as the transfer function of the dispersive system into account. Next, $g_{1}(E)$ is associated with the bulk plasmon in the sample with mean-free-path $\lambda_{1}$; the functions $g_{i}(E)$ represent different scattering processes with mean-free-path $\lambda_{i}$.

The computation of a spectrum profile requires a knowledge of the energy loss functions $g_{i}(E)$ associated with the various elemental processes as well as the transfer function $G_{\mathrm{s}}(E)$ to be defined.

\section{The Transfer Function}

The experimental energy loss spectra have been obtained with a Philips CM30 ST microscope and a PEELS Gatan 666 equipped with a YAG-photodiode parallel detection system, or with a Philips CM200 microscope coupled with the GIF device equipped with a parallel detection system using a CCD matrix. In the both cases, the linearity of the energy dispersion and the linearity of the response of the detection system have been verified.

The transfer function of the parallel detection systems has the form of a Gaussian function with a broadening of the feet of the curve. The transfer function profile depends on experimental conditions; we have chosen to model it as the experimental zero-loss peak detected with no sample in the same conditions as the spectra to be observed.

\section{The Main Elemental Process: Bulk Plasmon}

The first part of the spectrum is related to basic excitation processes of high probability and is interpreted in terms of multiple scattering. These primary excitation processes include interband transitions, surface and bulk plasmons, the latter being dominant. The dielectric function $\varepsilon$ is used to describe both single and collective excitations [27]. The energy loss function for a bulk plasmon can be written following the Drude model [28, 29]:

$$
\operatorname{Im}\left(-\frac{1}{\varepsilon}\right)=\frac{\frac{\omega}{\tau} \omega_{\mathrm{p}}^{2}}{\left(\omega^{2}-\omega_{\mathrm{p}}^{2}\right)^{2}+\frac{\omega^{2}}{\tau^{2}}} .
$$


$\omega_{\mathrm{p}}$ being the bulk plasmon frequency for collective excitation of free electrons; $\tau$ is a constant related to the life time of the excitation, which takes the damping of this excitation into account. By applying this dielectric function to a free electron gas, the single scattering profile $g_{1}(E)$ associated with the bulk plasmon process may be expressed as the normalized Drude function [25]:

$$
g_{1}(E)=K \frac{E}{\left(E^{2}-A^{2}\right)^{2}+4 B^{2} E^{2}}
$$

where $K$ is a normalization constant. The constants $A$ and $B$ are related to the most probable energy loss $E_{\mathrm{p}}=\hbar \omega_{\mathrm{p}}$ of the plasmon and to the life time $\tau$, i.e. to the full width at half maximum $\Delta E_{\mathrm{p}}$ of $g_{1}(E)$.

For some samples, a second inelastic process, which is assumed to arise from the collective excitation of the $\pi$ electrons [30-32], must be considered. It can be represented by a function $g_{2}(E)$ similar to $g_{1}(E)$, with a mean-free-path $\lambda_{2}$.

\section{K-Edge Profiles}

It is well established that the hydrogenic model and the Hartree-Slater model give good results for the evaluation of the $K$-shell excitation cross-section. A comparison of $K$-edge and crosssection profiles for low $Z$ elements [33] shows that the cross-section profiles describe the $K$-edge roughly for an edge where the fine structures are important, such as BN. In a first step, we have chosen to represent inner-shell profiles by the Hartree-Slater model (given in the Gatan $\mathrm{El} / \mathrm{P}$ software).

\section{Calculation Procedure}

The different excitation models are introduced in the convolution procedure following the equation (2) in Fourier space. In order to obtain the intensity as a function of the energy loss, an inverse Fourier transform is applied. After normalization by integrating over the total intensity, the parametrization procedure consists in the determination of the group of parameters giving the best fit of the calculated spectra to the experimental ones.

The initial values of the parameters are the theoretical or experimental values found in the literature. The best adjustment of the whole group of the parameters is performed by using the method of Levenberg-Marquardt [11]. Depending on whether the initial parameters are or are not far from the correct group of parameters, this technique uses one or other of two methods of minimization, a gradient or a linear method, givinga rapid convergence.

The first step in our calculation procedure is to determine the parameters of the dominant excitation process with accuracy. For that, we use the low loss part of spectrum up to the first characteristic edge.

The curves in Figure 1a represent the low-energy-loss part of boron nitride spectra corresponding to different thicknesses of the areas analysed. Each spectrum is obtained by taking two inelastic processes into account: the bulk plasmon centred on $26 \mathrm{eV}$ and a secondary process centred on $8 \mathrm{eV}$. The different parameters are adjusted by using the above method on the spectrum corresponding to the thinner part of the BN film. The parameters obtained are then introduced in the calculation for areas of increasing thickness. The parameter values for the fitting of the first part of BN spectra are collected in Table I, where $\rho$ is a coefficient that accounts for the quality of the fitting and which must be as small as possible. Here $\rho<0.03$ is considered as a limit value for a good fit. 

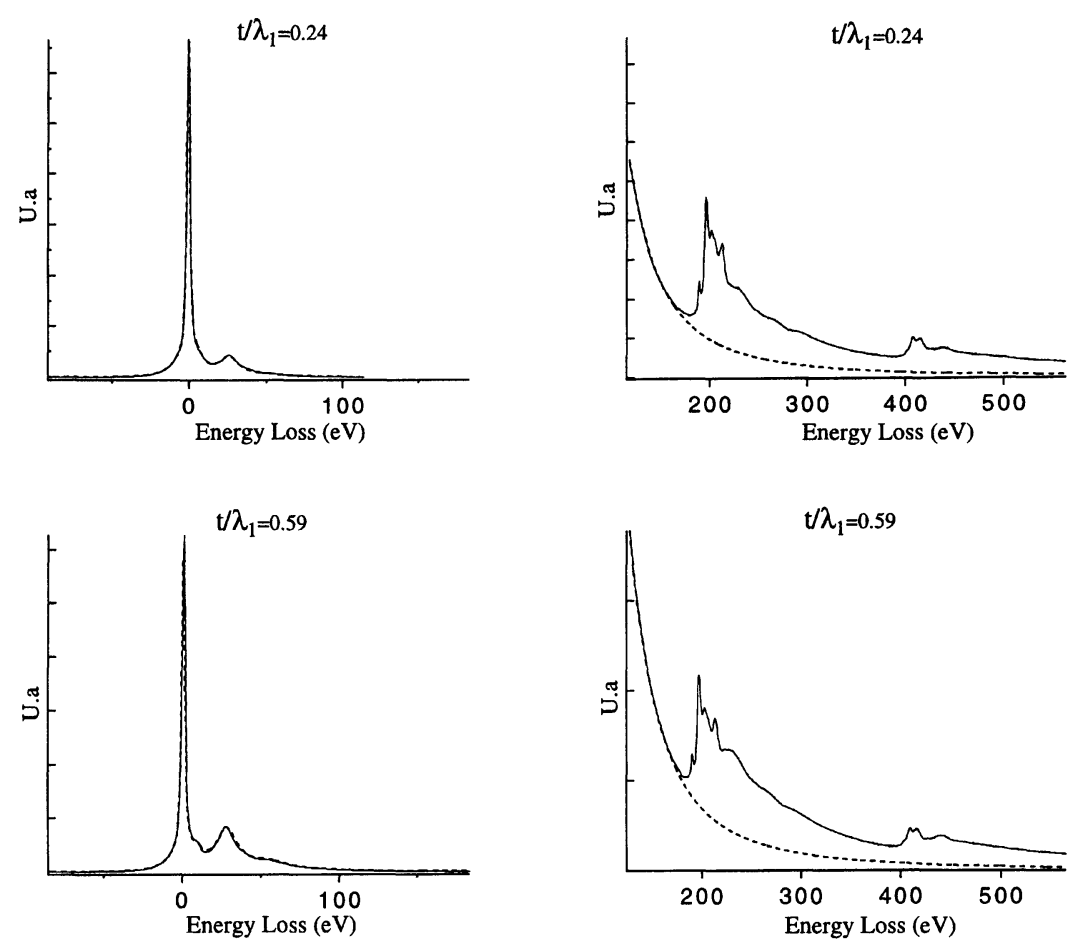

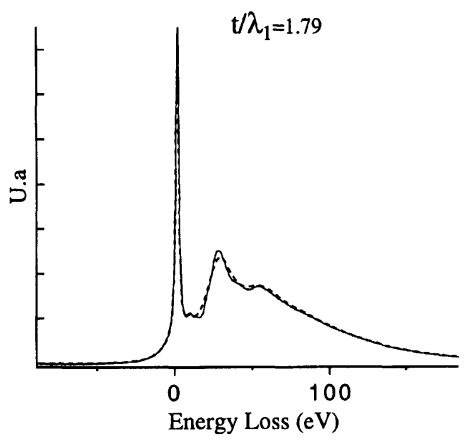

(a)

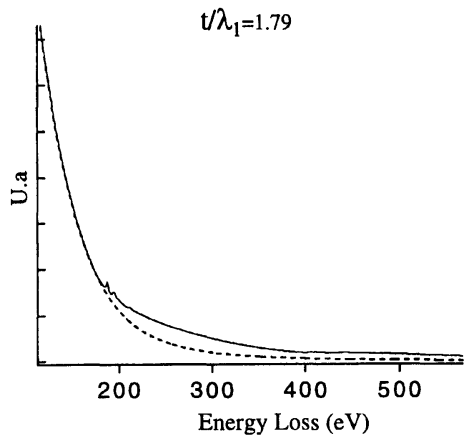

(b)

Fig. 1. - Boron nitride spectra: solid curves represent experimental data and dashed curves calculated spectra for the reduced thicknesses: $t / \lambda_{1}=0.24, t / \lambda_{1}=0.59$ and $t / \lambda_{1}=1.79$. The left curves (a) correspond to the low-loss part of spectra, while on the right curves (b) the dashed lines represent the calculated background under the $K$-edges of boron and carbon.

Table I. - Values of the parameters for the boron nitride low-loss part.

\begin{tabular}{|c|c|c|c|c|c|c|c|}
\hline & $t / \lambda_{1}$ & $E_{\mathrm{p} 1}$ & $\Delta E_{\mathrm{p} 1}$ & $E_{\mathrm{p} 2}$ & $\Delta E_{\mathrm{p} 2}$ & $\lambda_{1} / \lambda_{2}$ & $\rho$ \\
\hline $\mathrm{BN}-1$ & 0.27 & 26.5 & 12 & 8.7 & 4 & 0.11 & 0.017 \\
\hline $\mathrm{BN}-2$ & 0.59 & 26.3 & 12 & 8.7 & 4 & 0.09 & 0.020 \\
\hline $\mathrm{BN}-3$ & 1.79 & 26.5 & 11 & 8.7 & 4 & 0.13 & 0.034 \\
\hline
\end{tabular}


From one spectrum to another, only the thickness increases. These changes are taken into account by using the reduced thickness $t / \lambda_{1}$. The group of parameters $\left(E_{\mathrm{p} 1}, \Delta E_{\mathrm{p} 1}, E_{\mathrm{p} 2}, \Delta E_{\mathrm{p} 2}\right.$, $\left.\lambda_{1} / \lambda_{2}\right)$ determined for the thinner sample is also appropriate for the thicker areas, with an accuracy of the order of $10 \%$.

The misfit $\left\{I_{\exp }(E)-I_{\text {calc }}(E)\right\} / I_{\exp }(E)$ between the calculated and experimental profiles is less than $3 \%$, at least up to the energy loss corresponding to the first inner shell excitation edge appearing in the spectra as shown in Figure 1b. good fit demonstrates that the calculated background, when extrapolated below the characteristic distributions, can be used to strip the inner-shell distributions from the spectra when the "power-law" method fails, particularly for thick samples or when there is an overlap of the plasmon and characteristic distributions.

The second step in our procedure consists in the calculation of an entire spectrum, that is to say with the characteristic distributions. We have chosen two types of samples the spectra of which contain $K$-edges only: boron nitride and boron carbide. The $K$-edges have been calculated first by using the Hartree-Slater model for the cross-section profiles. The parts of the spectra containing $K$-edges are drawn in the Figure 2, after stripping from the background. These curves show that the calculated spectra reproduce the profiles of the experimental spectra except in the part of the fine structures of the $K$-edges where the misfit appears important. These results suggest that a $K$-edge model that allows this part of spectrum to be reproduced more acurately should be introduced.

In order to build a whole spectrum including $K$-edges by the convolution procedure, we have attempted to parametrize the energy loss functions associated with inner shell excitation processes in terms of simple analytical forms. The first step in our approach is to obtain the elementary distribution profiles from experimental spectra by using a deconvolution procedure (Fourier ratio deconvolution). Here the low-loss and the core-loss parts of the spectra must be recorded in the same experimental conditions. Before applying the deconvolution procedure, the background is removed from the characteristic distributions. We essentially use the analytical expression of the background for the $K$-edge stripping.

Two kinds of samples (metallic boron and amorphous carbon) and different thicknesses have been considered. After applying the deconvolution procedure, the $K$-edge profiles appear identical for each kind of sample, irrespective of the thickness. This confirms the validity of the deconvolution procedure. The deconvolved profiles from carbon and boron spectra are the same with an accuracy better than $5 \%$.

The experimentally deconvolved $K$-edge profiles for metallic boron and amorphous carbon are compared with the corresponding Hartree-Slater profiles in the curves of the Figures $3 \mathrm{a}$ and $3 \mathrm{~b}$.

In order to obtain a profile without noise or EXELFS modulations for example, the experimentally deconvolved $K$-edge can be parametrized in terms of an analytical form obtained by mixing a Gaussian function and a polynomial. The adjustment of the different parameters is optimized by using a refinement procedure based on a commonly used MLS method. This numerical model is obtained with the same values of the parameters for the boron and carbon $K$-edges.

By using the numerical models, spectra of BC3 of different thicknesses have been calculated: these are reproduced in the Figure 4. The fitting of the low-loss part of these spectra has been performed following the method described previously. The parameters, particularly the reduced thickness for each spectrum: $0.44,0.87,1.79$ are given in Table II. The agreement in all the cases is better than 7\%, only fine structures (such as ELNES and EXELFS) are not taken into account.

EELS quantitative elemental analysis can be performed from these calculated spectra by adjusting two weighting factors which are related to the number of atoms of each element. The 

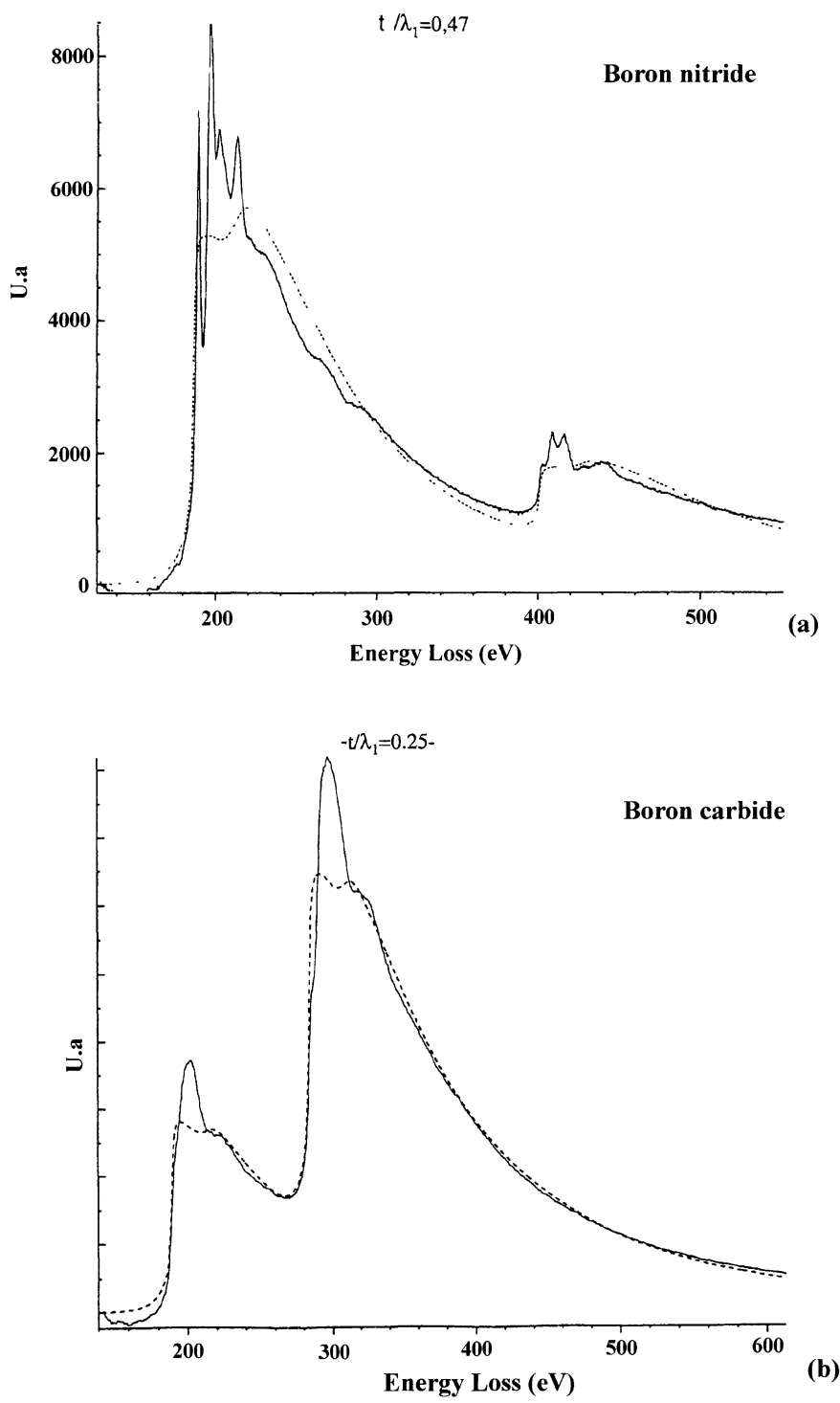

(a)

Fig. 2. - (a) Boron and nitrogen $K$-edges extracted from the spectrum of a boron nitride specimen, (b) boron and carbon $K$-edges extracted from the spectrum of a boron carbide specimen. Solid curves correspond to experimental spectra and dashed curves to calculated $K$-edges by using the HartreeSlater cross-section model.

Table II. - Values of the parameters for the boron carbide.

\begin{tabular}{|c|c|c|c|c|c|c|}
\hline & $t / \lambda_{1}$ & $E_{\mathrm{p} 1}$ & $\Delta E_{\mathrm{p} 1}$ & $\rho$ & $a_{\mathrm{B}} / a_{\mathrm{C}}$ & $N_{\mathrm{B}} / N_{\mathrm{C}}$ \\
\hline $\mathrm{BC}_{3}-1$ & 0.44 & 26.3 & 14.5 & 0.025 & 0.695 & 0.326 \\
\hline $\mathrm{BC}_{3}-2$ & 0.87 & 26.4 & 14.5 & 0.03 & 0.703 & 0.33 \\
\hline $\mathrm{BC}_{3}-3$ & 1.79 & 26.4 & 14.5 & 0.01 & 0.659 & 0.31 \\
\hline
\end{tabular}




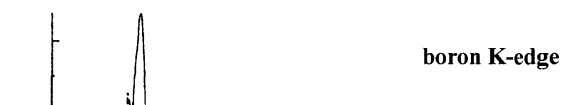

(a)
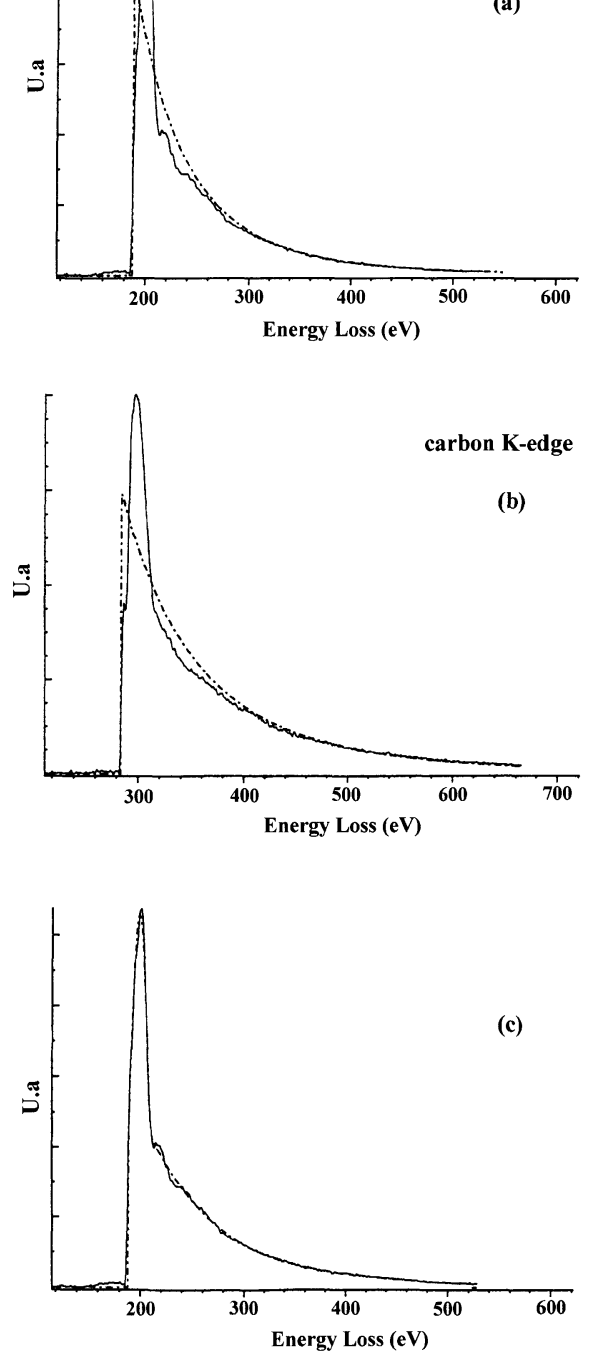

Fig. 3. - Deconvoluted $K$-edges of boron (a) and of carbon (b) compared to $K$-edge Hartree-Slater cross-section model. A template of $K$-edge (dashed curve) is superposed on the deconvoluted boron $K$-edge.

intensity $I\left(E, \theta_{\mathrm{c}}\right)$ of spectra above the first $K$-edge after background removal can be written in direct space $[17,34]$ :

$$
I\left(E, \theta_{\mathrm{c}}\right)=\Sigma_{i} a_{i} I_{\mathrm{B}}\left(E, \theta_{\mathrm{c}}\right) g_{i}(E)
$$

where $I_{\mathrm{B}}\left(E, \theta_{\mathrm{c}}\right)$ is the intensity of the spectrum calculated in the whole energy loss range of the spectrum without characteristic edges, and $g_{i}(E)$ is the energy loss function associated to the $i$ th $K$-edge in the spectrum; $a_{i}$ represents the weighting factor for the characteristic distribution $i$. 

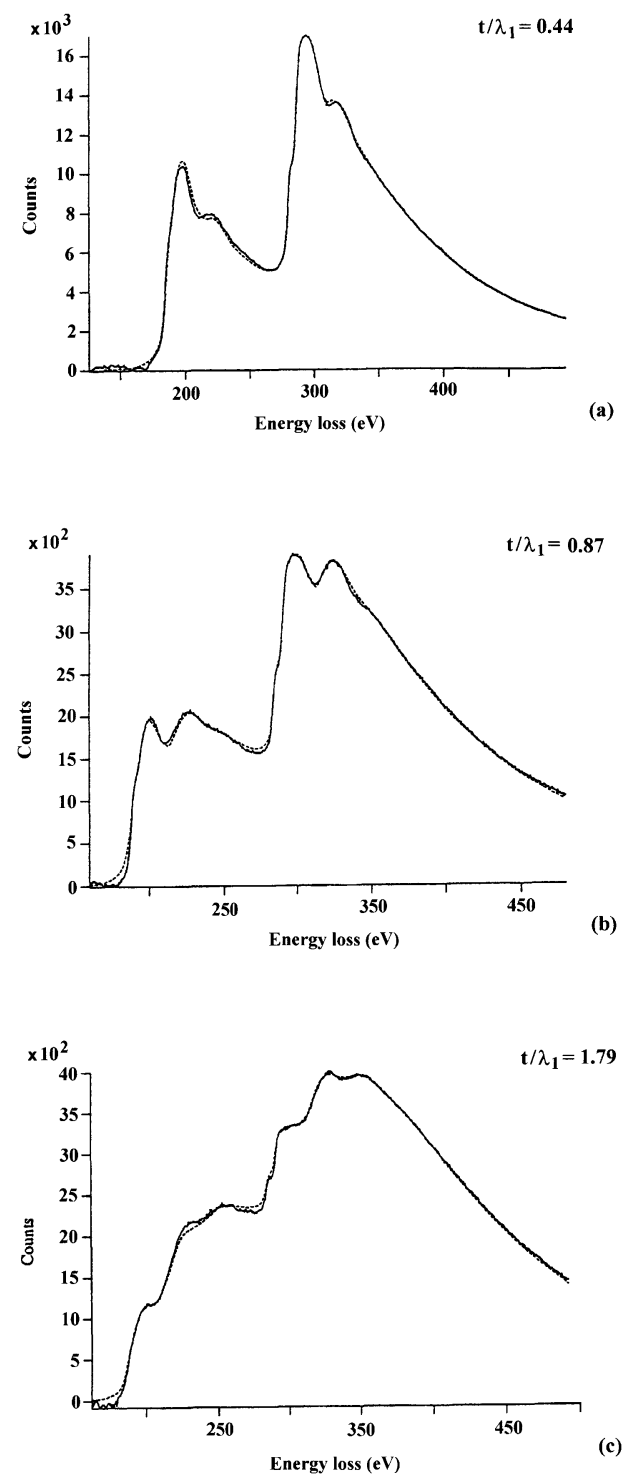

Fig. 4. - Boron and carbon $K$-edges of boron carbide spectra. Solid curves represent experimental data and dashed curves calculated spectra. (a) $t / \lambda_{1}=0.44$, (b) $t / \lambda_{1}=0.87$, (c) $t / \lambda_{1}=1.79$.

All the parameters concerning the first part of the spectra have been determined in the same way as in boron nitride.

$a_{\mathrm{B}} / a_{\mathrm{C}}$ are the ratios of boron to carbon weighting factors, and $N_{\mathrm{B}} / N_{\mathrm{C}}$ the atomic ratios that can be deduced from the building back method.

For quantitative analysis this technique gives good results for all the boron carbide samples. In the case of thin samples, the results are of the same order as those obtained from the classical method of stripping the $K$-edges. For thicker specimens, the power-law fails because of the overlap of the $K$-edges, while our technique allows to obtain an accuracy of the order of $6 \%$. 


\section{Conclusion}

These results confirm the validity of the parametrization method of electron energy loss spectra. They justify the theoretical models established in the literature, particularly for collective excitations and $K$-edge ionization processes. The background removal obtained by this method gives accurate results in quantitative analysis. The ionization process energy-loss functions obtained by a deconvolution procedure can be parametrized and used to build back a whole spectrum, in order to perform quantitative chemical analysis.

\section{References}

[1] Egerton R.F., Ultramicroscopy 3 (1978) 243.

[2] Egerton R.F., Electron Energy Loss Spectroscopy in the Electron Microscope (NewYork, Plenum Press, 1986).

[3] Egerton R.F., Microsc. Microanal. Microstruct. 2 (1991) 203.

[4] Stephens A.P., Ultramicroscopy 5 (1980) 343.

[5] Su D.S., Wang H.F. and Zeitler E., Ultramicroscopy 59 (1995) 181.

[6] Egerton R.F., Ultramicroscopy 4 (1979) 169.

[7] Hofer F., Microsc. Microanal. Microstruct. 2 (1991) 215.

[8] Hofer F., Kothleitner G. and Rez P., Ultramicroscopy 63 (1996) 239.

[9] Egerton R.F., Ultramicroscopy 50 (1993) 13.

[10] Egerton R.F., EELS in the Electron Microscope, 2nd Ed. (New York, Plenum Press, 1996).

[11] Bevington P.R., Data reduction and error analysis in the physical science (McGraw-Hill, New York, 1969), p. 105.

[12] Colliex C., Jeanguillaume C. and Trebbia P., Microprobe Analysis of Biological Systems, Hutchinson and Somlyo Eds. (Acad. Press, New york, 1981), p. 251.

[13] De Bruijn W., Ketelaars D., Gelsema E. and Sorber L., Microsc. Microanal. Microstruct. 2 (1991) 281.

[14] Wilson A.R., Microsc. Microanal. Microstruct. 2 (1991) 269.

[15] Tenailleau H and Martin J.M., J. Microscopy 166 (1992) 297.

[16] Shuman H. and Somlyo A.P., Ultramicroscopy 21 (1987) 23.

[17] Leapman R.D. and Swyt C.R., Ultramicroscopy 26 (1988) 393.

[18] Zaluzec N., Proc. 41st Ann. EMSA Meeting, G.W. Bailey Ed., p. 388, 1983.

[19] Craven A.J., Scott C.P., Gilmore C.J. and Bowen A.W., Proc. EMAG 91, Bristol, 105, 1988.

[20] Manoubi T., Tence M. and Colliex C., Ultramicroscopy 28 (1989) 49.

[21] Manoubi T., Tence M., Walls M.G. and Colliex C., Microsc. Microanal. Microstruct. 1 (1990) 23.

[22] Zanchi G., Kihn Y. and Sevely J., EUREM 88, Inst. Phys. Conf. Sci. 2 (1988) 177.

[23] Burge R.E. and Misel D.L., J. Phys. C 2 (1969) 1397.

[24] Wehenkel C. and Gauthe B., Phys. Stat. Sol. B 64 (1974) 515.

[25] Kihn Y., Thesis, Toulouse, 1985.

[26] Su D.S. and Zeitler E., Phys. Rev. B 47 (1993) 47.

[27] Ritchie R.H. and Howie A., Phil. Mag. 36 (1977) 463.

[28] Raether H., Springer Tracts in Modern Physics (Springer Verlag, 1980) p. 88.

[29] Pines D., Rev. Mod. Phys. 28 (1956) 184.

[30] Liang W.Y. and Cundy S.L., Phil. Mag. 19 (1968) 1031.

[31] Burge R.E. and Misell D.L., Phil. Mag. 18 (1968) 251.

[32] Zeppenfeld K., Z. Phys. 211 (1968) 391.

[33] Ahn C. and Rez P., Ultramicroscopy 17 (1985) 105.

[34] Door R. and Gangler D., Ultramicroscopy 58 (1995) 197. 